Bull. Austral. Math. Soc.

$47 \mathrm{H} 10,46 \mathrm{~B} 30$

VOL. 37 (1988) [81-87]

\title{
FIXED POINT OF SUM FOR CONCAVE AND CONVEX OPERATORS WITH APPLICATIONS
}

\author{
Li Bingyou
}

\begin{abstract}
In this paper we study fixed points of sums of $\alpha$-concave and $(-\alpha)$-convex operators in $\boldsymbol{\Upsilon}$-complete partially ordered linear spaces. As an application we obtain existence and uniqueness theorems for solutions of a certain type of nonlinear integral equation.
\end{abstract}

\section{INTRODUCTION}

The concept of $\alpha$-concave and $(-\alpha)$-convex operators was first introduced by Potter [5]. Then Guo Dajun, [1] studied fixed points and intrinsic elements of the two kinds of operators. Ortega [4] and Leggett [3] studied the fixed points of the sum and product of operators. In this paper we extended the real partially ordered Banach spaces in [5], [1] to $\Upsilon$-complete partially ordered linear spaces, and we study the fixed points of the sum of $\alpha$-concave and $(-\alpha)$-convex operators. We use the above result to obtain an existence and uniqueness theorem for the solution of a kind of nonlinear integral equation. Obviously the results in this paper are more general than those in $[\mathbf{3}]$ and $[\mathbf{5}]$.

\section{MaIN Results}

DEFINITION 1: Let $P$ be a positive cone in a $\Upsilon$-complete partially ordered linear space $E$ (see [2]). $\Phi$ is the set of interior points of $P$. An operator $f: \Phi \rightarrow \Phi \quad(0<$ $\alpha<1$ ) is called $\alpha$-concave (or $(-\alpha)$-convex) if it satisfies the following condition:

$$
\left.f(t x) \geqslant t^{\alpha} f x \quad \text { (or } f(t x) \leqslant t^{\alpha} f x\right) \quad \forall x \in \Phi, \quad 0<t<1
$$

It is easy to see that $f$ is $\alpha$-concave (or $(-\alpha)$-convex) if and only if

$$
\left.f(s x) \leqslant s^{\alpha} f x \quad \text { (or } f(s x) \geqslant s^{-\alpha} f x\right), \quad \forall x \in \Phi, \quad s>1
$$

Received 5 March 1987

Copyright Clearance Centre, Inc. Serial-fee code: $0004-9729 / 88 \$ A 2.00+0.00$. 
Theorem 1. Let $P$ be a positive cone in a $\Upsilon$-complete partially ordered linear space $E . g, h: \Phi \rightarrow \Phi$ are the increasing $\alpha$-concave and decreasing $(-\alpha)$-convex operators respectively, $0<\alpha<1$. Then the operator

$$
A x=g x+h x+C \quad(x \in \Phi, C \in P)
$$

has a unique fixed point $x^{\star}$ in $\Phi$, and for any $x_{0} \in \Phi$, we have

$$
x^{\star}=\vee\left\{x_{n}\right\}=\wedge\left\{x_{n}\right\}
$$

where $x_{n}=A x_{n-1}$, and we have the estimate

$$
0 \leqslant x^{\star}-x_{n} \leqslant\left(1-S_{0}^{-2 \alpha^{n}}\right) S_{0} x_{0}
$$

where

$$
S_{0}=\max \left\{S_{1}, S_{2}\right\}
$$

$$
\begin{aligned}
& S_{1}=\sup \left\{S>1: S^{\alpha-1} x_{0} \leqslant g x_{0}+h x_{0}\right\} \\
& S_{2}=\inf \left\{S>1: g x_{0}+h x_{0} \leqslant S^{1-\alpha} x_{0}\right\}
\end{aligned}
$$

Proof: First let $c=0$. Then it is clear that $S_{0}>1$. For any $x_{0} \in \Phi$, from [4] we have

$$
\frac{1}{2} S_{0}^{\alpha-1} x_{0} \leqslant g x_{0}+h x_{0} \leqslant \frac{1}{2} S_{0}^{1-\alpha} x_{0}
$$

Let $U_{0}=S_{0}^{-1} x_{0}, V_{0}=S_{0} x_{0}$ then $V_{0}>>U_{0}$. Put

$$
U_{n}=g U_{n-1}+h V_{n-1}, \quad V_{n}=g V_{n-1}+h U_{n-1} .
$$

We may prove by induction that

$$
\left[U_{n}, V_{n}\right] \subseteq\left[U_{n-1}, V_{n-1}\right], \quad(n=1,2, \ldots)
$$

Since $E$ is $\Upsilon$-incomplete, there exists $u^{\star}, v^{\star} \in E$ such that

$$
u^{\star}=\vee\left\{u_{n}\right\}, \quad v^{\star}=\wedge\left\{v_{n}\right\}
$$

and $u_{n} \leqslant u^{\star} \leqslant v^{\star} \leqslant v_{n}$. Thus

$$
u_{n-1}=g u_{n}+h v_{n} \leqslant g u^{\star}+h v^{\star} \leqslant g v_{n}+h u_{n}=v_{n+1} .
$$


Hence

$$
u_{n} \leqslant u^{\star} \leqslant g u^{\star}+h v^{\star} \leqslant v^{\star} \leqslant v_{n} .
$$

By induction it is easy to prove that

$$
u_{n} \geqslant S_{0}^{-2 \alpha^{n}} v_{n} \quad(n=0,1,2, \ldots) .
$$

From (8) and (9) we have

$$
0 \leqslant v^{\star}-u^{\star} \leqslant v_{n}-u_{n} \leqslant\left(1-S_{0}^{-2 \alpha^{n}}\right) v_{0} .
$$

By the Archimedean property we deduce that $v^{\star}=u^{\star}$. So by (8) it follows that $u^{\star}$ is a fixed point of $A$.

Now we prove uniqueness. Suppose $\bar{x}, \overline{\bar{x}} \in \Phi$ are two distinct fixed points of $A$. Then there exists $\mu>1$, such that

$$
\mu^{-\alpha} \bar{x} \leqslant \overline{\bar{x}} \leqslant \mu^{-\alpha} \bar{x}
$$

We may prove by induction that

$$
\mu^{-\alpha^{\prime \prime}} \bar{x} \leqslant \overline{\bar{x}} \leqslant \mu^{\alpha^{\prime \prime}} \bar{x}
$$

In the foregoing inequality we take the limit as $n \rightarrow \infty$ and obtain $\bar{x} \leqslant \overline{\bar{x}} \leqslant \bar{x}$. So $\bar{x}=\overline{\bar{x}}$.

Next we prove that $x^{\star}$, defined by (2), is a fixed point of $A$. Hence it is a unique fixed point. First, we may prove by induction that

$$
u_{n} \leqslant x_{n} \leqslant v_{n} \quad(n=0,1,2, \ldots) .
$$

Let $x_{\star}=\wedge\left\{x_{n}\right\}, x^{\star}=\vee\left\{x_{n}\right\}$. From (10) and (7), we obtain

$$
u_{n} \leqslant v^{\star} \leqslant x_{\star} \leqslant x^{\star} \leqslant v^{\star} \leqslant v_{n} .
$$

Since $u^{\star}=v^{\star}$, so $x_{\star}=x^{\star}=u^{\star}$. Hence $x^{\star}$ is a fixed point of $A$. By (11) and (9), we know that (3) is true. Finally, let $c \neq 0$. Since $G x=g x+\frac{1}{2} C, H x=h x+\frac{1}{2} C^{\prime}$ are increasing $\alpha$-concave and decreasing $(-\alpha)$-convex operators respectively, so the theorem is still valid in the case $c \neq 0$. 


\section{APPLICATIONS}

From Theorem 1 we obtain immediately:

ThOEREM 2. Under the conditions of Theorem 1, the equation

$$
B x=g\left(\frac{1}{x}\right)+h\left(\frac{1}{x}\right)+C=x
$$

has a unique solution.

THEOREM 3. Under the conditions of Theorem 1, we use $x_{\lambda}$ to denote the unique solution of the equation $A x=g x+h x+C=\lambda x$. Then $x_{\lambda}$ is decreasing for $\lambda$ (that is, $0<\lambda_{1}<\lambda_{2} \Rightarrow x_{\lambda_{1}}>x_{\lambda_{2}}$ ), o-continuous (that is, for $\lambda_{0}>0,0-\lim m_{\lambda \rightarrow \lambda_{0}} x_{\lambda}=x_{\lambda_{0}}$ ) and

$$
0-\lim _{\lambda \rightarrow+\infty} x_{\lambda}=0, \quad 0-\lim _{\lambda \rightarrow 0^{\star}} x_{\lambda}=+\infty \quad \text { (the infinite element in } \Phi .
$$

Proof: For $\lambda>0$, by Theorem 1 we know that $A x=\lambda x$ has a unique solution $x_{0}$ in $\Phi$. Let $0<\lambda_{1}<\lambda_{2}$, if $x_{\lambda_{2}} \& x_{\lambda_{2}}$, put

$$
\begin{aligned}
& M=\inf \left\{u: x_{\lambda_{2}} \leqslant u x_{\lambda_{1}}\right\} \\
& m=\sup \left\{\Theta: \Theta x_{\lambda_{1}} \leqslant x_{\lambda_{2}}\right\}
\end{aligned}
$$

It is easy to see that $M>1$ and $m>1$ and

$$
m x_{\lambda_{1}} \leqslant x_{\lambda_{2}} \leqslant M x_{\lambda_{1}}, \quad m \leqslant M
$$

If $m^{-1} \geqslant M^{-1}$, then $m \leqslant M^{-1}$. This contradicts $m>1$. Hence, $m^{-1}<M$, i.e., $M^{-1}<m$. Thus we have

$$
\begin{gathered}
M^{-1} x_{\lambda_{1}} \leqslant x_{\lambda_{2}} \leqslant M x_{\lambda_{1}} \\
x_{\lambda_{2}} \leqslant \frac{1}{\lambda_{2}}\left[g\left(M x_{\lambda_{1}}\right)+h\left(M^{-1} x_{\lambda_{1}}\right)+C\right] \leqslant \frac{\lambda_{1}}{\lambda_{2}} M^{\alpha} x_{\lambda_{1}} .
\end{gathered}
$$

By (13) we have $M=\frac{\lambda_{1}}{\lambda_{2}} M^{\alpha}$. Thus $\lambda_{2}<\lambda_{1}$. This contradicts the hypothesis of the theorem. Hence $x_{\lambda_{2}} \leqslant x_{\lambda_{1}}$. Since the fixed point of $\frac{1}{\lambda} A$ is unique, so $x_{\lambda_{2}}<x_{\lambda_{1}}$.

Now we prove $a$-continuity. We observe that $0<\lambda_{1}<\lambda_{2} \Rightarrow x_{\lambda_{2}}<x_{\lambda_{1}}$. Put

$$
\begin{aligned}
& m=\sup \left\{\Theta: \Theta x_{\lambda_{1}} \leqslant x_{\lambda_{2}}\right\} \\
& M=\inf \left\{\mu: x_{\lambda_{2}} \leqslant \mu x_{\lambda_{1}}\right\}
\end{aligned}
$$

It is easy to see that $0<m<1$,

$$
m x_{\lambda_{1}} \leqslant x_{\lambda_{2}} \leqslant M x_{\lambda_{1}}, \quad m \leqslant M
$$


If $m^{-1}<M$, then $M>1, M^{-1}<m$. Hence

$$
\begin{gathered}
M^{1} x_{\lambda_{1}} \leqslant x_{\lambda_{2}} \leqslant M x_{\lambda_{1}}, \\
x_{\lambda_{2}} \leqslant \frac{1}{\lambda_{2}}\left[g\left(M x_{\lambda_{1}}\right)+h\left(M^{-1} x_{\lambda_{1}}\right)+C\right] \leqslant \frac{\lambda_{1}}{\lambda_{2}} M^{\alpha} x_{\lambda_{1}} .
\end{gathered}
$$

By (14) we have

$$
M \leqslant \frac{\lambda_{1}}{\lambda_{2}} M^{\alpha}, \quad \frac{\lambda_{1}}{\lambda_{2}} \geqslant M^{1-\alpha}>1
$$

so $\lambda_{1}>\lambda_{2}$, which contradicts our hypothesis. Hence $M \leqslant m^{-1}$. Then we have

$$
m x_{\lambda_{1}} \leqslant x_{\lambda_{2}} \leqslant m^{-1} x_{\lambda_{1}}
$$

$$
x_{\lambda_{2}} \geqslant \frac{1}{\lambda_{2}}\left[g\left(m x_{\lambda_{1}}\right)+h\left(m^{-1} x_{\lambda_{1}}\right)+C\right] \geqslant \frac{\lambda_{1}}{\lambda_{2}} m^{\alpha} x_{\lambda_{1}}
$$

By (14) we have

$$
\frac{\lambda_{1}}{\lambda_{2}} m^{\alpha} \leqslant m, \quad\left(\frac{\lambda_{1}}{\lambda_{2}}\right)^{\frac{1}{1-\alpha}} \leqslant m .
$$

Hence

$$
\begin{gathered}
x_{\lambda_{2}} \geqslant m x_{\lambda_{1}} \geqslant\left(\frac{\lambda_{1}}{\lambda_{2}}\right)^{\frac{1}{1-\alpha}} x_{\lambda_{1}} \\
0<x_{\lambda_{1}}-x_{\lambda_{2}} \leqslant x_{\lambda_{1}}-\left(\frac{\lambda_{1}}{\lambda_{2}}\right)^{\frac{1}{1-\alpha}} x_{\lambda_{1}}=\left[1-\left(\frac{\lambda_{1}}{\lambda_{2}}\right)^{\frac{1}{1-\alpha}} x_{\lambda_{1}} .\right.
\end{gathered}
$$

In this inequality, let $\lambda_{1}=\lambda_{0}, \lambda_{2}=\lambda$. Then $x_{\lambda}$ is $o$-continuous with respect to $\lambda$. As in (15) we have

$$
\left(\frac{\lambda_{1}}{\lambda_{2}}\right) M^{-\alpha} x_{\lambda_{1}} \leqslant x_{\lambda_{2}}
$$

Hence

$$
\frac{\lambda_{1}}{\lambda_{2}} M^{-\alpha} x_{\lambda_{1}} \leqslant x_{\lambda_{2}} \leqslant \frac{\lambda_{1}}{\lambda_{2}} M^{\alpha} x_{\lambda_{1}}
$$

In this inequality, let $\lambda_{2}=\lambda$, and we see that (12) holds.

Theorem 4. Let $E$ be a $\Upsilon$-complete Riesz space of Banach type and $\Phi$ be a non-empty positive cone of $E$. With operator $A$ defined as in Theorem 1 , we have that $A$ is a contraction on $\Phi$. That is, there exists $r, R(0<r<R)$. such that

$$
\begin{gathered}
\forall x \in \Phi, \quad 0 \leqslant\|x\|<r \Rightarrow A x \not x, \\
\forall x \in \Phi, \quad\|x\|>R \Rightarrow A x \not x .
\end{gathered}
$$


Proof: By Theorem 1 we deduce that $A$ has a fixed point $x^{\star} . \forall x \in \Phi$, put

$$
\begin{aligned}
t_{0} & =\sup \left\{t: t x^{\star} \leqslant x\right\} \\
s_{0} & =\inf \left\{s: x \leqslant s x^{\star}\right\} .
\end{aligned}
$$

Obviously,

$$
t_{0} x^{\star} \leqslant x \leqslant s_{0} x^{\star}, \quad t_{0} \leqslant s_{0} .
$$

First we prove that

$$
x \in \Phi, \quad x \geqslant A x \Rightarrow x \geqslant x^{\star} \text {. }
$$

By (17), $s_{0}^{-1} \& t_{0}$. Hence $s_{0}<t_{0}^{-1}$. By (17), we have

$$
t_{0} x^{\star} \leqslant x \leqslant t_{0}^{--1} x^{\star}
$$

If $t_{0}<1$, then $x \geqslant A x \geqslant t_{0}^{\alpha} x^{\star}$. By (16), we have $t_{0}^{\alpha} \leqslant t_{0}$, which is a contradiction. So $t_{0} \geqslant 1$, and (18) holds.

Similarly, we have

$$
x \in \Phi, x \leqslant A x \Rightarrow x \leqslant x^{\star} .
$$

Since the interval in a $\Upsilon$-complete Riesz space of Banach type is bounded, from (18), (19) we see that the Theorem holds.

Theorem 5. Consider the integral equation

$$
\lambda x(t)=\int_{R^{n}}\left\{k_{1}(t, s) \sum_{i=1}^{\infty} a_{i}(s)[x(s)]^{\alpha_{i}}+k_{2}(t, s) \sum_{i=1}^{\infty} b_{i}(s)[x(s)]^{-\beta_{i}}\right\} d s
$$

where $\lambda>0, R^{n}$ is an $n$-dimensional Euclidean space. If

(i) $\alpha_{i}, \beta_{i}>0$ and $\sup _{i} \alpha_{i}=\sup _{i} \beta_{i}=\alpha>1$;

(ii) $k_{i}(\ell, s)(i=1,2)$ are nonnegative measureable functions on $R^{2 n}$, and there exist constants $m, M(0<m<M)$ such that.

$$
m \leqslant \int_{R^{n}} k_{i}(\ell, s) d s \leqslant M, \quad i=1,2, \quad \forall t \in R^{n}
$$

(iii) $a_{i}(s), b_{i}(s)$ are nonnegative measurable functions on $R^{n}$ and there exist constants $\Upsilon_{i}, \Theta_{i}(i=1,2), 0<\Theta_{i}<\Upsilon_{i}$ such that

$$
\Theta_{i} \leqslant \sum_{i=1}^{\infty} a_{i}(s) \leqslant \Upsilon, \quad \Theta_{2} \leqslant \sum_{i=1}^{\infty} b_{i}(s) \leqslant \Upsilon_{2}
$$

then equation (20) has a unique continuous solution $x_{\lambda}(t)$ satisfying the condition

$$
0<\inf _{t \in R^{n}} x_{\lambda}(t) \leqslant \sup _{t \in R^{n}} x_{\lambda}(t)<+\infty .
$$

ProOF: The proof is an easy application of Theorem 1. 


\section{REFERENCES}

[1] Guo Dajun, "'Fixed point and intrinsic element of a kind of concave and convex operator', science Bulletin (in Chinese) 30 (1985), 1132-1135.

[2] E. Hille and R.S. Phillips, "Functional Analysis and Semi-groups", American Mathematical Society Colloquium Publications 31 (1957).

[3] R.W. Leggett, "On certain nonlinear integral equations', J. Math. Anal. Appl. 57 (1977), $462-468$.

[4] J.M. Ortega and W.C. Rheinboldt, Iterative Solutions of Nonlinear Equations (Academic Press New York, 1970).

[5] A.J.B. Potter, "Application of Hilbert's projective metric to certain classes of non-homogenous operators"', Quart. J. Math. Oxford (2) 28 (1977), 93-99.

Department of Mathematics,

Hebei Teacher's University,

SHIJAIZHUANG,

China 